Research Article

\title{
Free Vibration Analysis of Arbitrary-Shaped Plates Based on the Improved Rayleigh-Ritz Method
}

\author{
Wenjie Guo $\mathbb{i}$ and Qingsong Feng $(\mathbb{D}$ \\ Engineering Research Center of Railway Environment Vibration and Noise Ministry Education, East China Jiaotong University, \\ Nanchang 330013, China
}

Correspondence should be addressed to Qingsong Feng; fqshdjtdx@aliyun.com

Received 9 May 2019; Accepted 9 August 2019; Published 17 October 2019

Academic Editor: Giosuè Boscato

Copyright (c) 2019 Wenjie Guo and Qingsong Feng. This is an open access article distributed under the Creative Commons Attribution License, which permits unrestricted use, distribution, and reproduction in any medium, provided the original work is properly cited.

\begin{abstract}
In this investigation, an improved Rayleigh-Ritz method is put forward to analyze the free vibration characteristics of arbitraryshaped plates for the traditional Rayleigh-Ritz method which is difficult to solve. By expanding the domain of admissible functions out of the structural domain to form a rectangular domain, the admissible functions of arbitrary-shaped plates can be described conveniently by selecting the appropriate admissible functions. Adopting the spring model to simulate the general boundary conditions, the problems of vibration of the arbitrary plate domain can be solved perfectly. Then, a numerical method is introduced to figure out the structure strain energy, kinetic energy, and elastic potential energy of the boundary. Finally, comparing the result with the simulation results and reference examples, the accuracy and convergence of this method are testified. Therefore, an effective new method is proposed for the guidance of the related research and practical engineering problems.
\end{abstract}

\section{Introduction}

Plate structures are widely used in various engineering fields such as civil engineering, aerospace, and vehicle engineering. Vibration of plates has extensively been investigated for many years. Analytical methods are firstly proposed to handle the vibration characteristic analysis of the plates of single shapes and simple boundary conditions. However, the problems of plates in practical engineering are usually more complex. Thus, research on plates of arbitrary shape with complicated boundary conditions is of great significance.

So far, scholars mainly utilize numerical methods to solve the vibration problems of the arbitrary-shaped plate and shell, for instance, finite element approach [1], generalized differential quadrature finite element method [2], general higher-order equivalent single layer theory [3], differential quadrature method $[4,5]$, complex variable methods [6], and differential volume method [7]. Ahmad et al. [8] proposed a method to overcome the disadvantage of former ways when approximate geometric structure and the influence of shear displacement were ignored, by using the curved thick shell finite element method. Gorman [9] proposed a method, the method of superposition, for solving the problems of free vibration analysis of the completely free rectangular plate in 1978. By using the method of superposition, solutions can satisfy identically the differential equation and boundary conditions with any desired degree of accuracy. Eigenvalues of four-digit accuracy are provided for a wide range of plate aspect ratios and modal shapes. Fantuzzi et al. [10] proposed a generalized differential quadrature finite element method (GDQFEM) for fourparameter functionally graded cracked plates of arbitrary shape. It is very convenient to solve the problems of arbitrary-shaped plates and shells with numerical methods because of their natural advantages [11]. Yet there are also many shortcomings. For instant, in terms of the finite element method, when dividing the grid, the accuracy is of vital importance on the user's experience, which takes so much energy to ensure the calculation precision. In addition, when the model parameters and dimensions are changed, it is necessary to remodel, which is time-consuming and laborious. 
Thus, an analytical method, as a simple and effective analysis method, is widely used in the static and dynamic analysis of simple plate and shell problems. Xie et al. [12] used the wave-based method to analyze the free and forced vibration of the elastically coupled annular plate. Brischetto [13] analyzed the convergence of the free vibration analysis solution of $3 \mathrm{D}$ equilibrium equations for plates and shells by using the exponential matrix method. There are also many analysis methods used for solving problems of plates [1416]. The Rayleigh-Ritz method is one of the most important analysis methods. Using the Rayleigh-Ritz method, Abrate [17] studied the vibration of point supported triangular plates. Karunasena and Kitipornchai [18], based on the Rayleigh-Ritz method and Reissner-Mindlin plate theory, analyzed free vibration of shear deformable general triangular plates with arbitrary combinations of boundary conditions. Pavlovic and Mbakogu [19] used the Rayleigh method to study the fundamental frequencies of vibration of circular plates. The Rayleigh-Ritz method is so widely used for solving problems of plates. Due to the inherent advantages such as easy to be combined with other methods, the analytical method is widely used. It can also reveal the structural vibration characteristics theoretically and facilitate the analysis of the mechanism. Furthermore, it can be used to test the numerical methods. So, the study of analytical methods is also of great significance. However, for the plate with complex shape, the test function is very difficult to obtain and the formula derivation is very complex. For these reasons, the traditional Rayleigh-Ritz method or other analytic methods are difficult to solve the vibration problems of arbitrary-shaped plates.

In order to solve the problems of arbitrary-shaped plates, one of the most crucial difficulties to overcome is how to deal with the boundary conditions of a curved edge. There are many methods to simulate complex boundary conditions nowadays such as high-order precision mixed finite element method, extended Kantorovich method [20], length undetermined method, and boundary integral equation method [21]. But these methods are computationally complex, and furthermore, when the boundary conditions are changed, mostly it is necessary to rederive the formula, which is quite tedious. In particular, when dealing with the boundary of curved edges, it is even more difficult. As a very flexible method, the method of spring simulation boundary is widely used, and the accuracy of this approach has been well validated $[22,23]$. The main idea of the method is to translate the boundary condition constraints into the solving of the elastic potential energy. Various boundary conditions are simulated by changing the corresponding stiffness values of linear springs and angle springs distributing along the edges linearly of the structure. By converting the boundary constraint into elastic potential energy, the influence of different boundary conditions on the vibration of the structure is analyzed. However, studies above mainly simulated the boundary of the straight edges, and there is less research on the boundary conditions of the curved edges.

Based on the Rayleigh-Ritz method, a semianalytic method called the improved Rayleigh-Ritz method is proposed to solve the free vibration problems of arbitraryshaped plates, which is an improvement and expansion of the Rayleigh-Ritz method. The traditional Rayleigh-Ritz method and some methods currently proposed to calculate the vibration of specific shape plates are not very adaptable, and many kinds of shapes of plates are too difficult to find suitable admissible functions to solve the vibration problems. The improved Rayleigh-Ritz method can be used in the calculation of vibration of arbitraryshaped plates. It is suitable for various solutions without renewing the model while complex boundary conditions are changed, which is of higher efficiency and adaptability. The main innovation of the present method is that, on the one hand, the domain of admissible functions can be extended to the outside of the structure, and then the admissible functions can be used to simulate the arbitraryshaped plates' displacement. On the other hand, combining the spring model method, the present method can be used to solve problems of complex boundary conditions especially on the curved edges. The basic solving process of the present method is basically the same as that of the Rayleigh-Ritz method, which turns the vibration problem of plates into the eigenvalue problem. In addition, the method of this paper is more convenient in programming and analysis. Especially when the model size, materials, or boundary conditions of the structure are changed, there is no need to rederive the formula or build the model, and by just changing the parameters, the problems can be solved easily, which is quite time-saving and labor-saving. Compared with the traditional Rayleigh-Ritz method, this method has a larger application range and higher precision. It provides an effective reference for solving the problems of arbitrary-shaped plates in engineering problems.

\section{The Improved Rayleigh-Ritz Method}

The physical model of an arbitrary-shaped thin plate is shown in Figure 1. The structural domain $S$ is of arbitrary shape, and the springs are adopted to simulate general boundary conditions, linear springs and rotational springs. The spring constants are $k$ and $K$, respectively.

\subsection{Main Features of the Method}

2.1.1. Expansion of the Admissible Function Domain. When using the improved Rayleigh-Ritz method, the admissible function domain is expanded to the outside of the real structure, and the model is shown in Figure 2.

$S$ is the structural domain. And $S_{a}$ is the integral domain of the admissible function, the length is $L_{x}$ and $L_{y}$, respectively. When the structural domain is of arbitrary irregular shape, we can suppose that the structural domain can be extended to a rectangular domain slightly larger than the real domain of the structure, and the rectangular domain must contain the real structural domain. According to the rectangular domain, the admissible function can easily be obtained. By integrating according to the original structural domain, the strain energy and 


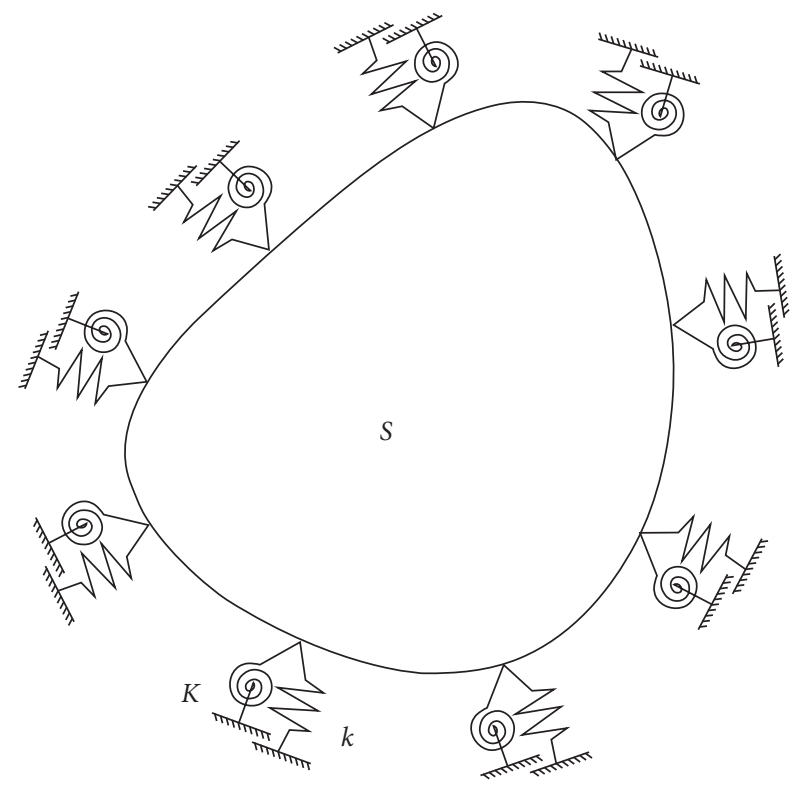

Figure 1: Physical model of an arbitrary-shaped thin plate with springs.

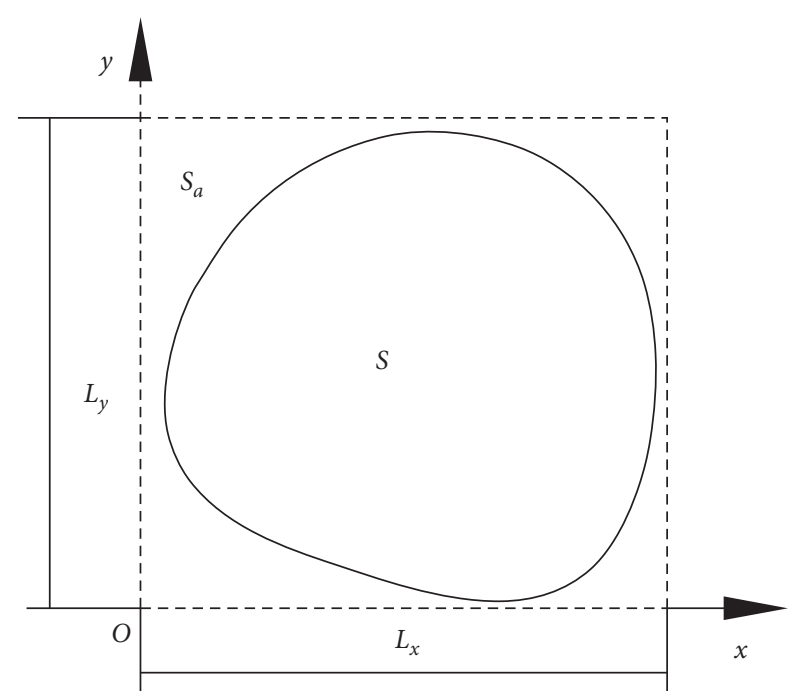

FIGURE 2: The relationship between integral domain and structural domain.

kinetic energy of the plate are obtained, and the calculation results are very accurate. The closer the admissible function domain is to the structural domain, the more accurate the solution is. So, a principle of the method to select the admissible function domain is to keep the rectangular domain as small as possible. Therefore, when the rectangular field is tangent to the irregular structure, the error is minimized.

When obtaining the strain energy and kinetic energy of the plate, integration needs to be performed. Set a circular plate whose radius is $R$ as an example. Due to its symmetry, when calculating the integral, only a quarter of the structure needs to be calculated, and the bending strain energy is

$$
\begin{aligned}
V_{R}= & \frac{1}{2} D \int_{0}^{R} \int_{0}^{\sqrt{R^{2}-y^{2}}}\left[\left(\frac{\partial^{2} w(x, y)}{\partial^{2} x}\right)^{2}+\left(\frac{\partial^{2} w(x, y)}{\partial^{2} y}\right)^{2}\right. \\
& +2 \mu\left(\frac{\partial^{2} w(x, y)}{\partial^{2} x}\right) \cdot\left(\frac{\partial^{2} w(x, y)}{\partial^{2} y}\right) \\
& \left.+2(1-\mu)\left(\frac{\partial^{2} w(x, y)}{\partial x \partial y}\right)^{2}\right] \mathrm{d} x \mathrm{~d} y, \\
T_{R}= & \frac{\rho h}{2} \int_{0}^{R} \int_{0}^{\sqrt{R^{2}+y^{2}}}\left(\frac{\partial w}{\partial t}\right)^{2} \mathrm{~d} x \mathrm{~d} y .
\end{aligned}
$$

Then, the strain energy and kinetic energy of the circular plate can be obtained by double integral. Normally, the edges of the irregular shape can be fitted by a polynomial, and the energy can be obtained by the integral. It is important to notice that when calculating these forms of double integral calculation, it is very difficult to solve some complicated functions by direct integral. In order to solve this problem, and improve the efficiency of calculation as well, we introduced the discrete method to calculate the above points. The specific way of discretization of the structural domain is shown in Figure 3.

Along the $y$ direction, the entire integral domain is uniformly divided into $Q$ equal parts with width $\mathrm{V}_{y}$ and the corresponding $y$ coordinate $y_{j}$. According to the function expression of the curve, the corresponding length of the integral domain can be calculated as

$$
L x_{j}=x_{R}-x_{L} .
$$

Then, the above length is divided into $Q$ parts, and the length of the small segment after discretization is

$$
\mathrm{V} x_{j}=\frac{L x_{j}}{\mathrm{Q}} .
$$

The horizontal and vertical coordinates of the discrete subsections are

$$
\begin{gathered}
x_{i j}=L x_{j}+(i-0.5) \cdot \mathrm{V} x_{j}, \\
y_{i}=y_{\text {min }}+(i-0.5) \cdot \mathrm{V} y .
\end{gathered}
$$

Therefore, the double integral can approximately be converted into the sum of the microarea, so the strain energy and kinetic energy can be expressed as follows:

$$
V_{c}=C \sum_{i=1}^{Q} \sum_{j=1}^{Q} f\left(x_{i j}, y_{i}\right) \cdot \mathrm{V} x_{j} \cdot \mathrm{V} y,
$$

where $C$ is the coefficient and $f\left(x_{i j}, y_{i}\right)$ is the related function of calculating strain energy and kinetic energy.

In this way, not only the computational efficiency is greatly improved, but also problems with some complicated integral functions can be solved. 


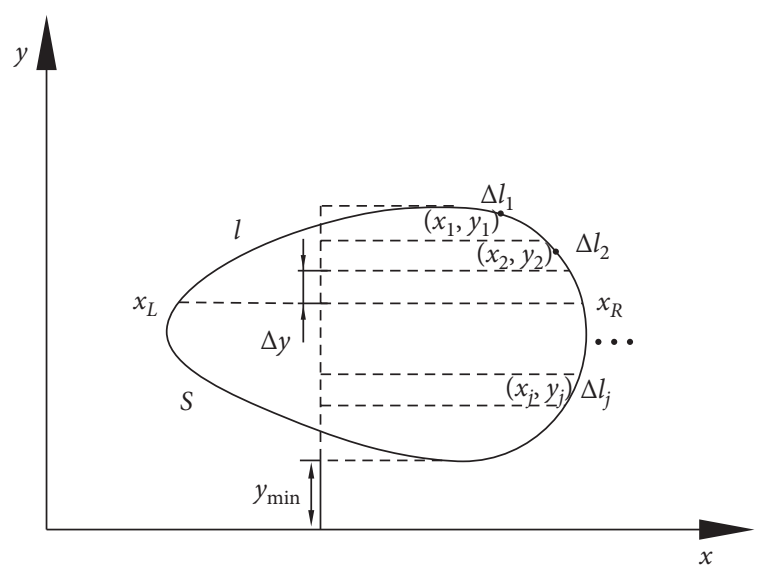

FIGURE 3: Diagram of the discrete model along the vertical axis.

2.1.2. Virtual Spring Model Method Handling the Curved Boundary Conditions. Classical boundary conditions, such as fixed and simple support, are often difficult to accurately simulate the boundary conditions of the actual structure. Considering the general condition, the spring model is used to simulate the more general boundary conditions. On the edges of the structural domain, the linear springs and the rotational springs are adopted. Set the rectangular plate as an example, the model is shown in Figure 4.

Supposing the linear spring constants and the rotational spring constants are $k_{i j}\left(\mathrm{~N} / \mathrm{m}^{2}\right)$ and $K_{i j}(\mathrm{~N} / \mathrm{rad})$, respectively. It is easy to simulate various complex boundary conditions by adjusting the stiffness of the two kinds of springs. The simulation of classical boundary conditions is described below. The specific constants are shown in Table 1 .

The mechanism of the spring model method is to transform the influence of the boundary conditions on the structural vibration to the increase of elastic potential energy of the total stiffness of the structure.

For the arbitrary-shaped plate studied in this paper, its edges are curved, and then the elastic potential energy of boundary is expressed as

$$
V_{s}=\frac{1}{2} \int_{l}\left[k w(x, y)^{2}+K\left(\frac{\partial w(x, y)}{\partial n^{V}}\right)^{2}\right] \mathrm{d} l,
$$

where $V_{s}$ is the spring elastic potential energy, $w \cdot(x, y)$ is the admissible function of the thin plate, $n^{V}$ is the normal direction of the edge, and $l$ is the total length of the edge, as shown in Figure 5.

The partial derivative of the curve in the normal direction is expressed as

$$
\frac{\partial w(x, y)}{\partial n^{V}}=\frac{\partial w(x, y)}{\partial x} \cos \theta+\frac{\partial w(x, y)}{\partial y} \sin \theta .
$$

Discretize the edge, as shown in Figure 6.

The length of each microsegment is

$$
\Delta l_{j}=\sqrt{\Delta y^{2}+\Delta x_{j}^{2}}
$$

Then, the elastic potential energy at the boundary can be expressed as

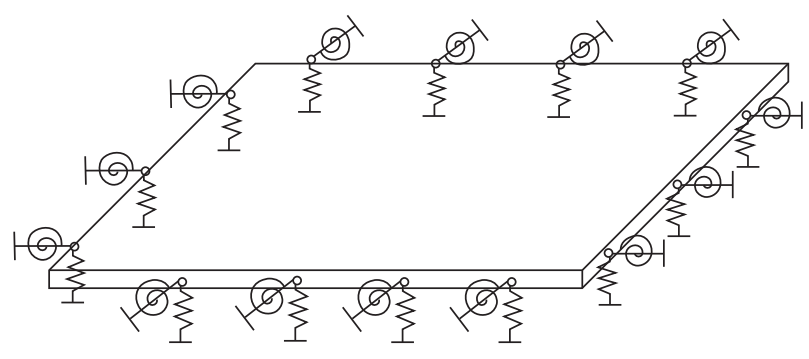

FIGURE 4: Model of the rectangular thin plate.

TABle 1: Classical boundary conditions corresponding spring values.

\begin{tabular}{lccc}
\hline & Clamped & Simply supported & Free \\
\hline$k\left(\mathrm{~N} / \mathrm{m}^{2}\right)$ & $\infty$ & $\infty$ & 0 \\
$K(\mathrm{~N} / \mathrm{rad})$ & $\infty$ & 0 & 0 \\
\hline
\end{tabular}

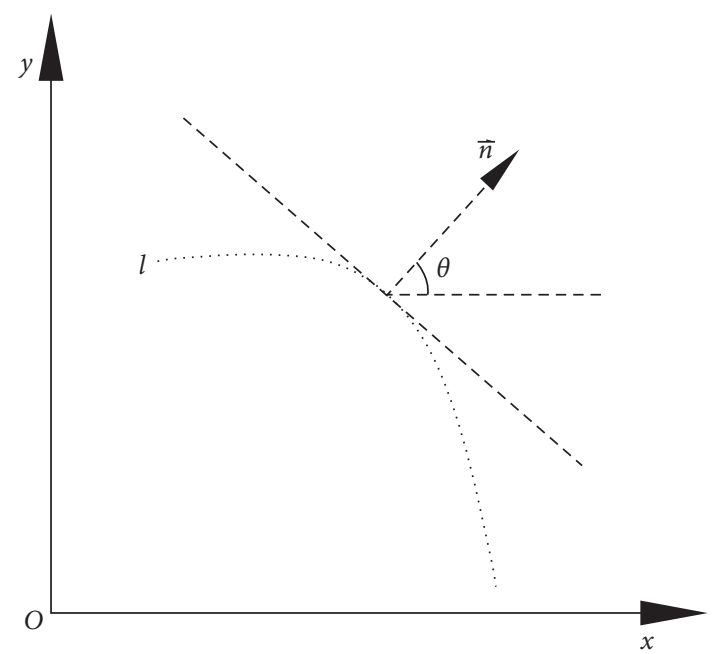

FiguRE 5: Border partial derivative calculation diagram.

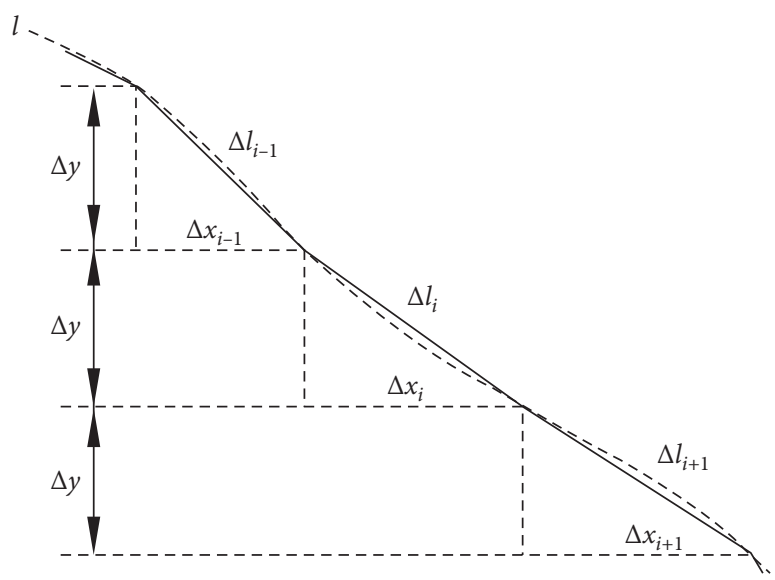

Figure 6: Diagram of discrete boundary edges. 


$$
V_{s c}=\frac{1}{2} \sum_{j=1}^{Q}\left[k w\left(x_{j}, y_{j}\right)^{2}+K\left(\left.\frac{\partial w(x, y)}{\partial n^{\mathrm{V}}}\right|_{x=x_{j}, y=y_{j}}\right)^{2}\right] \Delta l_{j} \text {. }
$$

2.1.3. Analysis Process. The admissible function of the thin plate can be expressed as

$$
w(x, y, t)=\sum_{m=1}^{M} \sum_{n=1}^{N} A_{m n} f_{m}(x) g_{n}(y) e^{i w t},
$$

where $A_{m n}$ is the unknown expansion coefficient, $m$ and $n$ are the serial numbers, $M$ and $N$ are the truncated items, $f_{m}(x)$ and $g_{n}(y)$ are the orthogonal polynomials along the $x$ and $y$ directions, Chebyshev series is selected, and $e^{i \omega t}$ is the simple harmonic time factor.

It is very important to select the appropriate admissible function when applying the improved Rayleigh-Ritz method for it has a great influence on the accuracy because the Chebyshev series can satisfy arbitrary boundary conditions and satisfy the condition that its third-order derivative is continuous and the fourth-order derivative exists at each point, which can overcome the disadvantage that the phenomenon of discontinuity may appear at the boundary. The present method chooses the Chebyshev series as admissible function [24,25] as follows:

$$
\begin{aligned}
& f_{1}(x)=1, \\
& f_{2}(x)=\frac{x}{L_{x}} \\
& f_{m+2}(x)=\frac{2 x}{L_{x}} f_{m+1}(x)-f_{m}(x), \\
& g_{1}(y)=1, \\
& g_{2}(y)=\frac{y}{L_{y}}, \\
& g_{n+2}(y)=\frac{2 y}{L_{y}} g_{n+1}(y)-g_{n}(y),
\end{aligned}
$$

where $m=1,2,3, \ldots, M$ and $n=1,2,3, \ldots, N$.

In order to be able to calculate the vibration problem of plates with arbitrary shape in complicated boundary conditions, the admissible function chosen in the present method has the following characteristics: it is an orthogonal function satisfying the corresponding governing equation, there is arbitrariness at the boundary, that is, some geometric boundary conditions cannot be met naturally, and the boundary conditions are only related to the spring stiffness constant.

The bending strain can be expressed as

$$
\begin{aligned}
V_{p}= & \frac{1}{2} D \iint_{S}\left[\left(\frac{\partial^{2} w(x, y)}{\partial^{2} x}\right)^{2}+\left(\frac{\partial^{2} w(x, y)}{\partial^{2} y}\right)^{2}\right. \\
& +2 \mu\left(\frac{\partial^{2} w(x, y)}{\partial^{2} x}\right)\left(\frac{\partial^{2} w(x, y)}{\partial^{2} y}\right) \\
& \left.+2(1-\mu)\left(\frac{\partial^{2} w(x, y)}{\partial x \partial y}\right)^{2}\right] \mathrm{d} x \mathrm{~d} y
\end{aligned}
$$

where $\mu$ is called Poisson's ratio, $D=E h^{3} /\left(12\left(1-\mu^{2}\right)\right)$ is the flexural rigidity, and $h$ is the thickness of the plate.

Ignoring the mass of the springs at the edges, the kinetic energy of the structure is

$$
T=\frac{1}{2} \rho h \iint_{s}\left(\frac{\partial w(x, y)}{\partial t}\right)^{2} \mathrm{~d} x \mathrm{~d} y
$$

where $\rho$ is the mass density.

The energy functional of the system can be expressed as

$$
\prod=V_{p}+V_{s}-T
$$

Substituting formulas (6), (12), and (13) into equation (14), and taking the total energy for partial derivatives:

$$
\frac{\partial \prod}{\partial A_{m n}}=0
$$

where $A_{m n}$ is the unknown coefficient used to describe bending vibration of the thin plate.

Then, the vibration problem of the structure is transformed into the problem of solving eigenvalues, which can be expressed as follows:

$$
\left(\mathbf{K}-\omega^{2} \mathbf{M}\right) \mathbf{A}=\mathbf{0},
$$

where $\mathbf{K}$ is the stiffness matrix, $\mathbf{K}=\mathbf{K}_{s}+\mathbf{K}_{p}$, where $\mathbf{K}_{s}$ is the stiffness matrix of spring energy and $\mathbf{K}_{p}$ is the stiffness matrix of the overall structure strain energy. $\mathbf{M}$ is the mass matrix of the structure, $\mathbf{A}$ is the unknown coefficient vector, and $\omega$ is the circular frequency. In this way, the free vibration problems can be transformed into the eigenvalue problem.

\section{Numerical Analysis}

In this section, set the calculations of free vibration problems of rectangle, triangle, parallelogram, round, and oval thin plate as examples, and by comparing the results of the present method with the results of references and FEM, good convergence and accuracy of the present method are proved. The material parameters of the plate are as follows: Young's modulus is equal to $2.1 \times 10^{11} \mathrm{~Pa}$, Poisson's ratio is equal to 0.3 , and density is equal to $7850 \mathrm{~kg} / \mathrm{m}^{3}$.

3.1. Convergence. The values of truncated number $M$ and $N$ in the admissible function, the spring stiffness, and the discrete division $Q$ may have a great influence on the accuracy of the calculation via the present method. The convergence analysis mainly focuses on the abovementioned quantities. 
By choosing the calculations of free vibration of rectangular plates under simply supported and clamped boundary conditions as examples, the convergence of the improved Rayleigh-Ritz method is tested. The parameters of the physical model are as follows: the length is $a=2 \mathrm{~m}$, the width is $b=1 \mathrm{~m}$, and the thickness is $h=0.02 \mathrm{~m}$, as shown in Figure 7.

Convergence analysis is carried out on the truncated number $M$ and $N$ first. Table 2 shows the first 10 natural frequencies of the plate changing with $M$ and $N$ when the number of division $Q$ is a constant and the stiffness coefficients of the springs equal to zero.

From the data in Table 2, the natural frequencies of the vibration of the rectangular plate tend to be fixed with the increase of the truncation terms $M$ and $N$. When $M=N=12$, the change of natural frequency is already very small, it can be considered that the method has converged.

The convergence of the division value $Q$ is illustrated. According to Table 2, the truncation terms $M$ and $N$ are equal to 12 , the stiffness coefficient is 0 , and the natural frequencies of the plate on different $Q$ are compared, as shown in Table 3.

From Table 3, the variation of natural frequencies of the rectangular plate is very small with increasing $Q$. When $Q$ is equal to 40 , the natural frequency is basically the same, so it can be thought that the present method has converged.

The convergence analysis is carried out on the stiffness of the spring next. Sometimes when simulating complex boundary conditions, the stiffness coefficients may need to be infinite such as simply supported or clamped conditions. However, when calculating by Matlab, the coefficients cannot be infinite actually. So, we choose a very large number instead. Table 4 shows the values of spring stiffness coefficient convergence tending to infinite. The values of the selected spring stiffness coefficient are related to the bending stiffness $D$. Suppose $k=p \mathrm{~N} / \mathrm{m}^{2}$ and $K=p \mathrm{~N} / \mathrm{rad}$.

From Table 4, we can conclude that the free vibration frequencies of the clamped plate tend to be a stable value as the stiffness coefficient increases gradually. When the stiffness coefficient is $k=10^{8} \mathrm{DN} / \mathrm{m}^{2}$ and $K=10^{8} \mathrm{DN} / \mathrm{rad}$, it converges. Therefore, when simulating complex boundary conditions and the stiffness of the spring is infinite, the stiffness value of the spring can be just $p=10^{8} \mathrm{D}$ to get an accurate solution.

\subsection{Accuracy}

3.2.1. Free Vibration Analysis of Rectangular Plate. To verify the accuracy of the present method, free vibration frequencies of rectangular plates are calculated. The parameters of the rectangular plate are length $a=1 \mathrm{~m}$, width $b=1 \mathrm{~m}$, and thickness $h=0.02 \mathrm{~m}$. Calculating the dimensionless frequency $\Omega=\omega a^{2} \sqrt{\rho h / D}$, the results are compared with reference [26] in Tables 5-7. The calculating formula for error is

$$
\text { Error }=\left|\frac{f_{\text {ref }}-f_{\text {present }}}{f_{\text {ref }}}\right| \times 100 \% .
$$

Tables 5-7 show that the present method calculated results are very close to the reference, which proves that

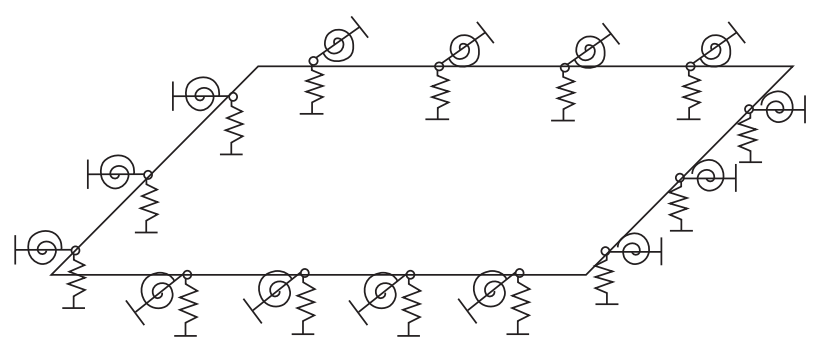

Figure 7: Physical model of the rectangular plate.

when calculating the free vibration of rectangular plates under complex boundary conditions, the present method is very accurate.

3.2.2. Free Vibration Analysis of Triangular Plate. To illustrate the accuracy of the method for solving the problems of free vibration on plates with hypotenuse edges under complex boundary conditions, natural frequencies of the triangular plate under different boundary conditions are obtained. The physical model is shown in Figure 8. The length of the two short sides is $a=1 \mathrm{~m}$, the length of the long side is $b=2 \mathrm{~m}$, and the thickness of the plate is $\mathrm{h}=0.02 \mathrm{~m}$.

In this section, two kinds of boundary conditions are selected for modal analysis of triangular plates, simply supported and elastic boundary (set $K=0 \mathrm{~N} / \mathrm{rad}$ ). Calculating the natural frequencies, and comparing with the results of FEM, the accuracy of the method can be proved in Tables 8-9 and Figures 9-10.

From Tables 8 and 9, comparing with the results of the finite element method, the natural frequency of the triangular plate via the present method is accurate and the error is very small. Figures 9 and 10 show the corresponding modes of the first four orders of the two methods. (the red part shows convex, and the blue part shows concave). As can be seen from the figure, the corresponding modes of the first four orders of the triangular plate are almost the same. It can be seen that the results of the present method are highly consistent with the FEM results, which proves the correctness and accuracy of the method when solving free vibration problems of plates with hypotenuse.

3.2.3. Free Vibration Analysis of Elliptical Plate. In order to verify the applicability of the present method for the curved edge plates under different boundary conditions, the vibration analysis of elliptical plates under different boundary conditions is discussed, as shown in Figure 11. The long axis of the ellipse model is $a=4 \mathrm{~m}$, short axis $b=2 \mathrm{~m}$, and thickness $h=0.02 \mathrm{~m}$.

In this section, two kinds of boundary conditions are selected, simply supported and clamped. By using the present method, the natural frequencies are obtained. Compared with the results of FEM, the accuracy of the method can be proved in Tables 10-11 and Figures 11-12.

From Tables 10 and 11, the natural frequencies of the elliptical plate calculated via the present method are in good agreement with the results of FEM, Figures 12 and 13 give the corresponding modes of the first four orders under the 
TABle 2: Natural frequencies of rectangular plate on different $M$ and $N(\mathrm{~Hz})$.

\begin{tabular}{lccccc}
\hline Mode & $M=N=8$ & $M=N=10$ & $M=N=12$ & $M=N=14$ & 26.84 \\
1 & 26.84 & 26.84 & 26.84 & $3=16$ \\
2 & 33.28 & 33.16 & 33.15 & 73.15 & 73.04 \\
3 & 73.23 & 73.07 & 73.05 & 74.74 & 73.15 \\
4 & 74.80 & 110.75 & 74.74 & 110.06 & 74.02 \\
5 & 110.11 & 127.15 & 110.06 & 127.08 & 110.05 \\
6 & 127.47 & 130.02 & 127.10 & 129.99 & 127.04 \\
7 & 130.13 & 149.23 & 130.00 & 149.21 & 129.97 \\
8 & 150.54 & 180.35 & 189.21 & 180.23 & 189.21 \\
9 & 180.91 & 201.37 & 201.25 & 201.21 & 201.16 \\
10 & 204.87 & & & \\
\hline
\end{tabular}

TABLE 3: Natural frequencies of the rectangular plate on different $Q(\mathrm{~Hz})$.

\begin{tabular}{lccccc}
\hline Mode & $Q=10$ & $Q=20$ & $Q=30$ & $Q=40$ & 26.76 \\
\hline 1 & 27.16 & 26.84 & 26.78 & 33.11 & 72.90 \\
2 & 33.05 & 33.15 & 33.12 & 74.37 & 33.11 \\
3 & 72.81 & 73.05 & 72.94 & 72.75 \\
4 & 76.15 & 74.74 & 74.46 & 109.73 & 126.60 \\
5 & 11.35 & 110.06 & 109.81 & 129.66 & 109.69 \\
6 & 126.62 & 127.10 & 126.73 & 148.21 & 126.54 \\
7 & 130.95 & 130.00 & 148.45 & 179.76 & 148.09 \\
8 & 153.09 & 149.21 & 179.90 & 199.98 & 179.70 \\
9 & 180.93 & 180.27 & 200.31 & & 199.82 \\
10 & 204.14 & 201.25 & & & \\
\hline
\end{tabular}

TABLE 4: Natural frequencies of the rectangular plate on different spring stiffness coefficients (Hz).

\begin{tabular}{|c|c|c|c|c|c|c|}
\hline Mode & $p=10^{5} \mathrm{D}$ & $p=10^{6} D$ & $p=10^{7} \mathrm{D}$ & $p=10^{8} D$ & $p=10^{9} \mathrm{D}$ & $p=10^{5} \mathrm{D}$ \\
\hline 1 & 121.97 & 122.15 & 122.17 & 122.17 & 122.17 & 121.97 \\
\hline 2 & 157.75 & 158.05 & 158.08 & 158.09 & 158.09 & 157.75 \\
\hline 3 & 221.55 & 222.10 & 222.15 & 222.16 & 222.16 & 221.55 \\
\hline 4 & 312.91 & 313.85 & 313.95 & 313.96 & 313.96 & 312.91 \\
\hline 5 & 316.46 & 317.64 & 317.76 & 317.77 & 317.77 & 316.46 \\
\hline 6 & 351.20 & 352.73 & 352.88 & 352.90 & 352.90 & 351.20 \\
\hline 7 & 410.91 & 413.06 & 413.27 & 413.30 & 413.30 & 410.91 \\
\hline 8 & 429.83 & 431.43 & 431.60 & 431.61 & 431.60 & 429.83 \\
\hline 9 & 496.51 & 499.59 & 499.90 & 499.93 & 499.93 & 496.51 \\
\hline 10 & 571.89 & 574.50 & 574.76 & 574.79 & 574.79 & 571.89 \\
\hline
\end{tabular}

TABLE 5: Natural frequencies of free vibration on rectangular plates under the condition of clamped edges $(\mathrm{Hz})$.

\begin{tabular}{lccc}
\hline Mode & & $\begin{array}{c}\Omega=\omega a^{2} \sqrt{\rho h / D} \\
\text { Ref. [26] }\end{array}$ & Error (\%) \\
\hline 1 & Present & 35.985 & 0.091 \\
2 & 35.952 & 73.395 & 0.105 \\
3 & 73.318 & 73.395 & 0.094 \\
4 & 73.326 & 108.22 & 0.114 \\
5 & 108.10 & 131.58 & 0.110 \\
6 & 131.44 & 131.20 & 0.610 \\
\hline
\end{tabular}

two boundary conditions (the red part of the figure shows convex, and the blue part is concave).

3.2.4. Free Vibration Analysis of Isosceles Trapezium Plate with Curved Sides. We combined the shapes above to verify the applicability of the present method in solving free vibration of complex-shaped thin plates, and we combined the following two kinds of borders: straight edges and curved edges. Take the trapezoid with curved sides as an example, and analyze the free vibration characteristics. The model is shown in Figure 14, in which No. 2 and No. 4 edges are curved. 
TABLE 6: Natural frequency of free vibration on the rectangular plate under the condition of one side clamped and three sides free (Hz).

\begin{tabular}{|c|c|c|c|}
\hline \multirow{2}{*}{ Mode } & \multicolumn{3}{|c|}{$\Omega=\omega a^{2} \sqrt{\rho h / D}$} \\
\hline & Present & Ref. [26] & Error (\%) \\
\hline 1 & 3.471 & 3.470 & 0.039 \\
\hline 2 & 8.508 & 8.504 & 0.045 \\
\hline 3 & 21.296 & 21.279 & 0.078 \\
\hline 4 & 27.217 & 27.201 & 0.059 \\
\hline 5 & 30.965 & 30.948 & 0.054 \\
\hline 6 & 54.219 & 54.185 & 0.063 \\
\hline
\end{tabular}

TABLE 7: Natural frequencies of the rectangular plate under the condition of two adjacent sides simply supported and others free (Hz).

\begin{tabular}{lccc}
\hline Mode & & $\begin{array}{c}\Omega=\omega a^{2} \sqrt{\rho h / D} \\
\text { Ref. [26] }\end{array}$ & 0.077 \\
\hline 1 & Present & 3.370 & 0.003 \\
2 & 3.367 & 17.321 & 0.021 \\
3 & 17.321 & 19.293 & 0.049 \\
4 & 19.297 & 38.203 & 0.057 \\
5 & 38.222 & 51.032 & 0.022 \\
6 & 51.061 & 53.497 & Error $(\%)$ \\
\hline
\end{tabular}

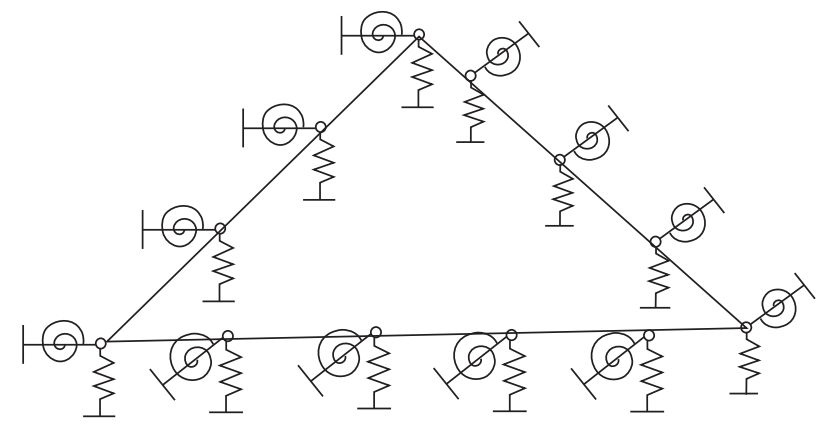

Figure 8: Triangular physical model.

TABLE 8: Natural frequencies of free vibration on the triangular plate under the boundary condition of three-side simply supported (Hz).

\begin{tabular}{lccc}
\hline Mode & & Simply supported & \\
& Present & FEM & 0.4576 \\
1 & 122.93 & 122.37 & 0.4344 \\
3 & 245.85 & 244.79 & 0.5079 \\
4 & 319.61 & 318.00 & 0.4072 \\
5 & 417.94 & 416.25 & 0.3997 \\
6 & 491.69 & 489.74 & 0.6723 \\
7 & 614.69 & 610.59 & 0.3358 \\
8 & 639.18 & 637.05 & 0.3489 \\
9 & 713.41 & 710.93 & 0.4398 \\
10 & 836.13 & 832.47 & 0.3938 \\
\hline
\end{tabular}

The parameters of the isosceles trapezoidal plate with curved sides in this section are as follows: the upper bottom length is $a=4 \mathrm{~m}$, the lower bottom length is $b=10 \mathrm{~m}$, the highest is $c=8 \mathrm{~m}$, the two hypotenuses are $1 / 4$ elliptic curve whose long axis is $8 \mathrm{~m}$ and the short axis is $3 \mathrm{~m}$, as shown in Figure 14. Tables 12-13 show the comparison of the natural frequencies of the first 10 orders. The natural frequencies of the trapezoidal plate are calculated by the present method and then compared with the FEM. The data show that the results of the two methods are in good agreement and the error is less than $1 \%$.

\section{Discussion}

The analysis above showed that the improved Rayleigh-Ritz method can be used to solve the free vibration problems of 
TABLE 9: Natural frequencies of the triangular plate under the condition of elastic boundary (Hz).

\begin{tabular}{|c|c|c|c|c|c|c|c|}
\hline \multirow{2}{*}{$k\left(\mathrm{~N} / \mathrm{m}^{2}\right)$} & \multicolumn{7}{|c|}{ Mode } \\
\hline & & 1 & 2 & 3 & 4 & 5 & 6 \\
\hline \multirow{3}{*}{$10^{6}$} & Present method & 37.63 & 92.79 & 149.08 & 204.17 & 256.77 & 365.43 \\
\hline & FEM & 37.35 & 92.24 & 148.06 & 202.88 & 255.07 & 362.61 \\
\hline & Error (\%) & 0.76 & 0.59 & 0.69 & 0.64 & 0.67 & 0.78 \\
\hline \multirow{3}{*}{$10^{7}$} & Present method & 81.44 & 133.47 & 175.81 & 228.84 & 276.61 & 379.54 \\
\hline & FEM & 80.86 & 80.86 & 80.86 & 80.86 & 80.86 & 80.86 \\
\hline & Error (\%) & 0.72 & 0.60 & 0.81 & 0.62 & 0.74 & 0.91 \\
\hline \multirow{3}{*}{$10^{8}$} & Present method & 117.03 & 219.36 & 281.84 & 343.33 & 398.05 & 483.37 \\
\hline & FEM & 116.35 & 218.15 & 279.74 & 341.69 & 395.28 & 478.54 \\
\hline & Error (\%) & 0.59 & 0.55 & 0.75 & 0.48 & 0.70 & 1.01 \\
\hline
\end{tabular}
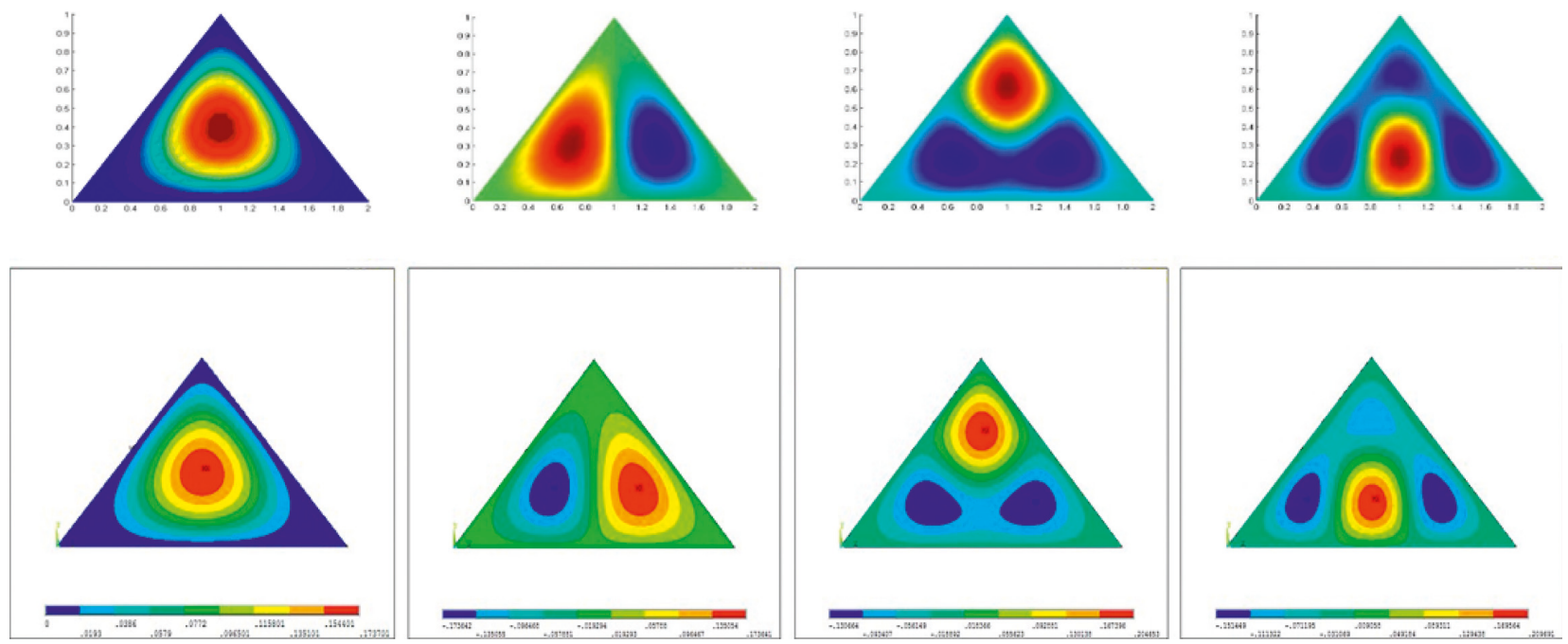

FIGURE 9: Comparison of the free vibration diagram of the corresponding modes of first four orders under the simply supported boundary condition.
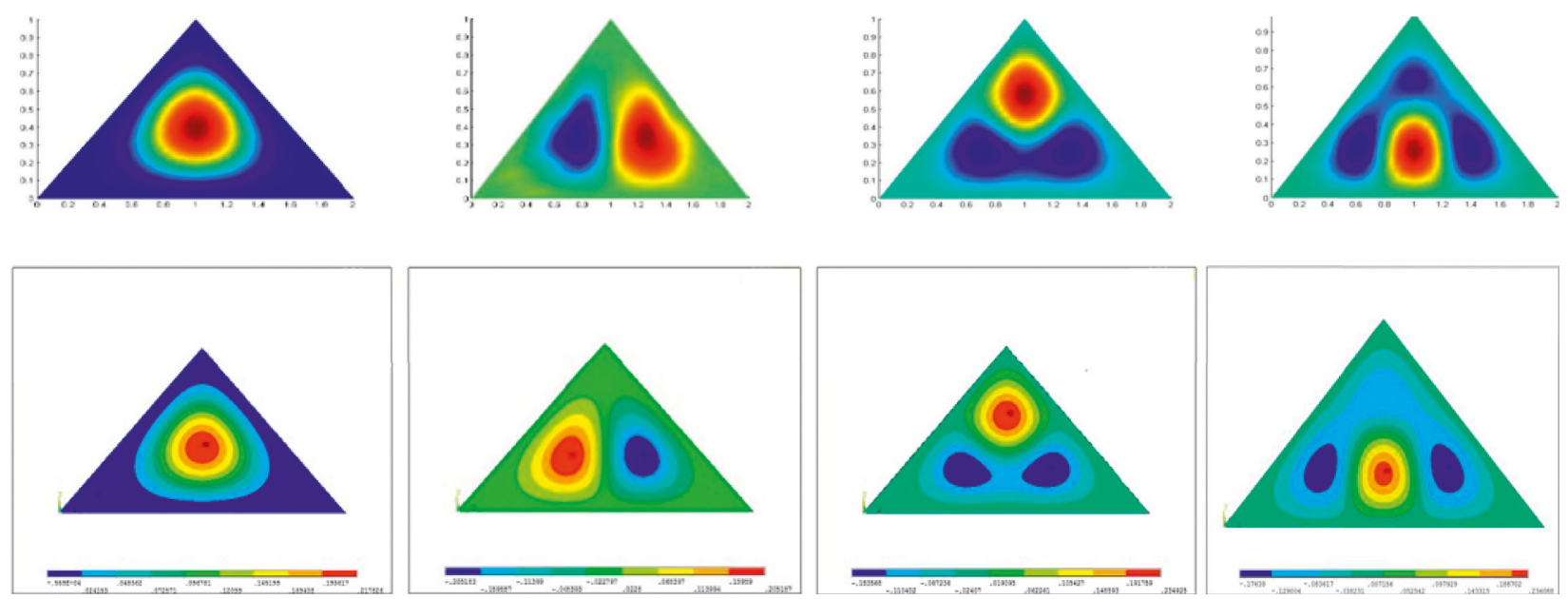

Figure 10: Comparison of the free vibration diagram of the corresponding modes of first four orders under the clamped boundary condition.

the arbitrary-shaped plates perfectly whose outer boundary is arbitrary. Yet, how about the inner boundary? The numerical example shows that this method is also applicable to the problems when the inner boundary is arbitrary. Set the rectangular plates with arbitrary-shaped center opening as an example to illustrate. 


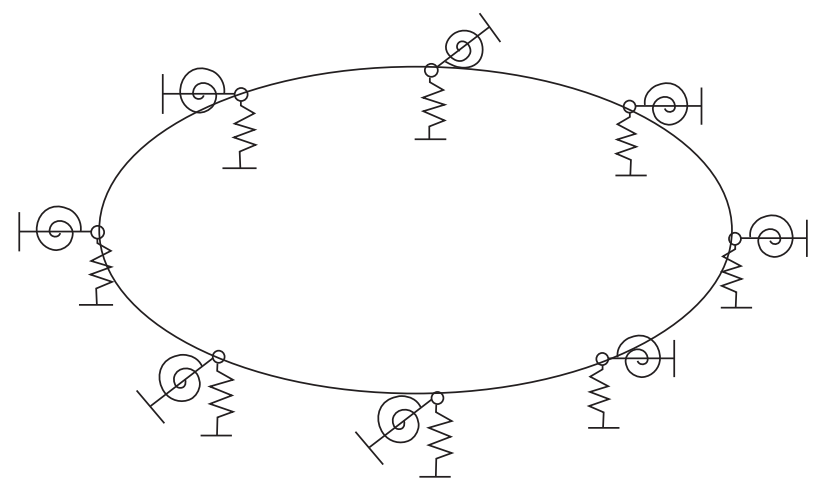

FIgUre 11: Physical model of the elliptical thin plate.

Table 10: Natural frequencies of the elliptical thin plate under the simply supported boundary condition $(\mathrm{Hz})$.

\begin{tabular}{lccc}
\hline Mode & & Simply supported & \\
& Present & FEM & 0.22 \\
1 & 16.45 & 16.49 & 0.22 \\
2 & 29.45 & 29.51 & 0.44 \\
3 & 47.69 & 47.90 & 0.95 \\
4 & 57.29 & 57.84 & 0.56 \\
5 & 71.60 & 72.00 & 0.65 \\
6 & 78.19 & 78.70 & 0.87 \\
7 & 101.01 & 101.91 & 1.03 \\
8 & 103.05 & 123.09 & 0.89 \\
9 & 121.99 & 134.59 & 0.88 \\
10 & 133.40 & & \\
\hline
\end{tabular}

TABle 11: Natural frequencies of the elliptical thin plate under the clamped boundary condition (Hz).

\begin{tabular}{lccc}
\hline Mode & & Clamped & \\
\hline 1 & Present & FEM & 0.08 \\
2 & 34.123 & 34.153 & 0.18 \\
3 & 49.200 & 49.292 & 0.24 \\
4 & 69.721 & 69.893 & 0.30 \\
5 & 87.090 & 87.359 & 0.34 \\
6 & 95.895 & 96.228 & 0.35 \\
7 & 109.697 & 110.09 & 0.51 \\
8 & 127.815 & 128.47 & 0.40 \\
9 & 136.944 & 137.5 & 0.83 \\
10 & 164.982 & 166.37 & 0.66 \\
\hline
\end{tabular}
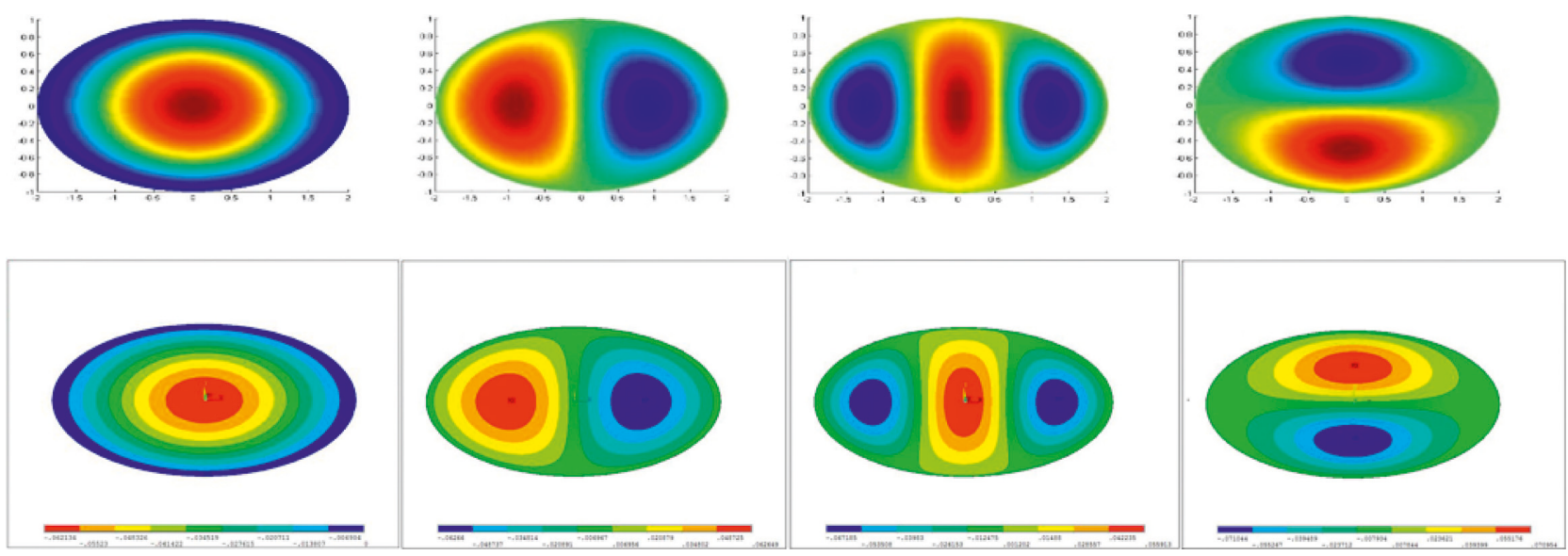

Figure 12: Comparison of the free vibration diagram of the corresponding modes of the first four orders under the simply supported boundary condition. 

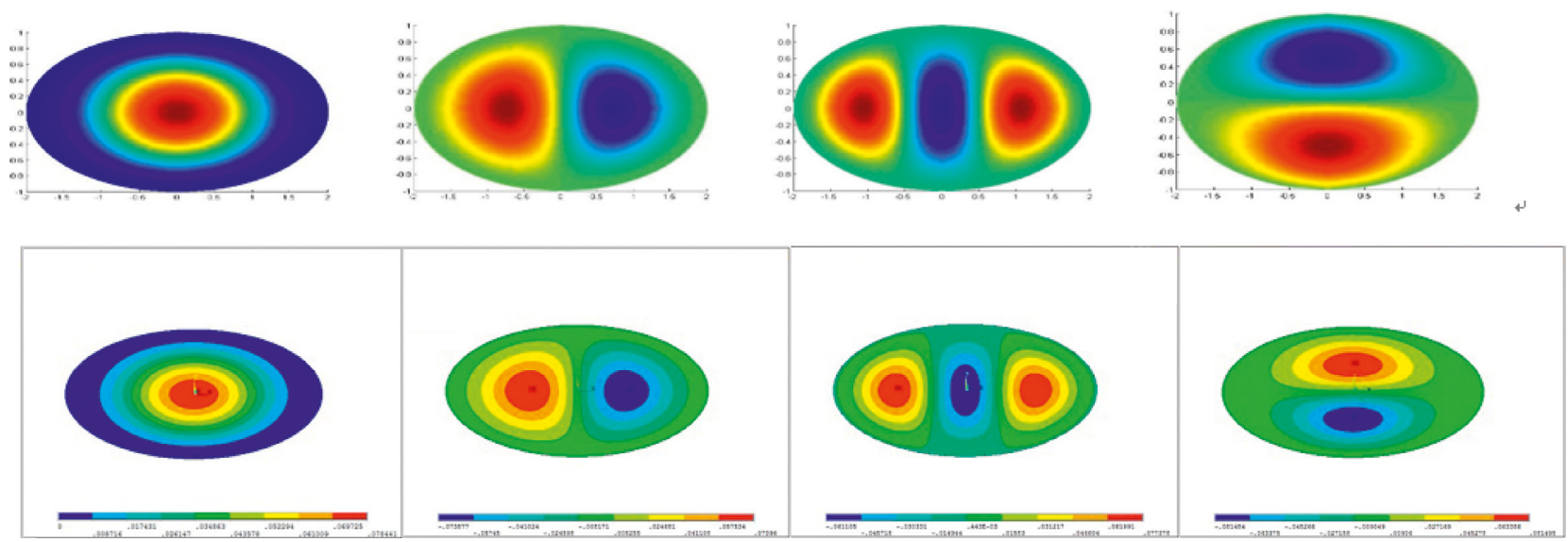

Figure 13: Comparison of the free vibration diagram of the corresponding modes of first four orders under the clamped boundary condition.

TABLE 12: Natural frequencies of the isosceles trapezoidal plate with curved sides under the clamped boundary condition (Hz) (C-C-C-C).

\begin{tabular}{lccc}
\hline Mode & & Clamped & \\
& Present & FEM & 0.50 \\
1 & 2.55 & 2.54 & 0.22 \\
2 & 4.77 & 4.76 & 0.36 \\
3 & 5.58 & 5.56 & 0.63 \\
4 & 7.91 & 7.86 & 0.15 \\
5 & 8.16 & 8.17 & 0.21 \\
6 & 10.08 & 10.06 & 0.05 \\
7 & 11.70 & 11.69 & 0.66 \\
8 & 12.44 & 12.36 & 0.08 \\
9 & 12.58 & 12.59 & 0.29 \\
10 & 15.63 & 15.68 & \\
\hline
\end{tabular}

TABLE 13: Natural frequencies of the isosceles trapezoidal plate under the simply supported boundary condition (S-S-S-S) (Hz).

\begin{tabular}{lccc}
\hline Mode & & Simply supported & \\
\hline 1 & Present & FEM & 0.70 \\
2 & 1.34 & 1.33 & 0.35 \\
3 & 3.15 & 3.14 & 0.33 \\
4 & 3.70 & 3.69 & 0.36 \\
5 & 5.70 & 5.68 & 0.17 \\
6 & 6.06 & 6.05 & 0.22 \\
7 & 7.52 & 7.51 & 0.19 \\
8 & 9.09 & 9.07 & 0.20 \\
9 & 9.58 & 9.56 & 0.16 \\
10 & 9.97 & 9.96 & 0.06 \\
\hline
\end{tabular}

In terms of the free vibration problems of rectangular plates with center openings, the method to select the displacement function and to simulate the complex boundary condition is almost the same as the plates analyzed above. What is different is that due to the perfect symmetry of the model, in order to simplify the calculation, only a quarter of the structure was studied, which can be seen in the study of the circular plate and ellipse plate as well. The compute model of the rectangular plate with circular opening is shown in Figure 15.
The strain energy of the plates of opening should be obtained by using the whole strain energy minus the strain energy of the opening, which is true of the kinetic energy. Take a rectangular plate with circular opening as an example to show the formulas to introduce it. Relevant geometric parameters are as follows: the length of rectangular plate is $a=6 \mathrm{~m}$, the width is $b=4 \mathrm{~m}$, the thickness is $h=0.02 \mathrm{~m}$, and the inner opening radius is $R=1 \mathrm{~m}$.

The bending strain energy of the rectangular plate without opening is expressed as 


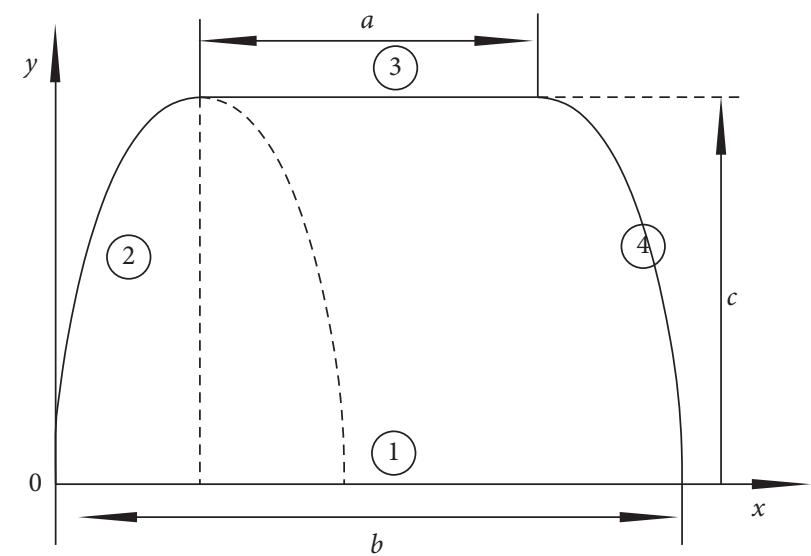

FIgURE 14: Isosceles trapezoidal plate with curved sides.

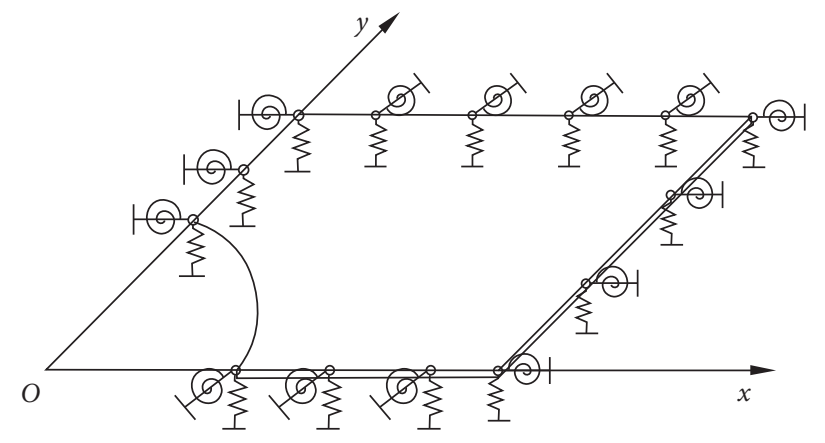

FIGURE 15: Compute model of the rectangular plate with circular opening.

TABLE 14: Natural frequencies of the rectangular plate with circular opening in C-C-C-C boundary (Hz) (C-C-C-C).

\begin{tabular}{lccc}
\hline Mode & & Clamped & \\
& Present & FEM & 0.08 \\
1 & 10.511 & 10.519 & 1.79 \\
2 & 12.189 & 11.975 & 0.27 \\
3 & 19.851 & 19.797 & 2.92 \\
4 & 23.837 & 23.162 & 0.28 \\
5 & 24.993 & 25.064 & 1.13 \\
6 & 31.751 & 31.397 & Error \\
\hline
\end{tabular}
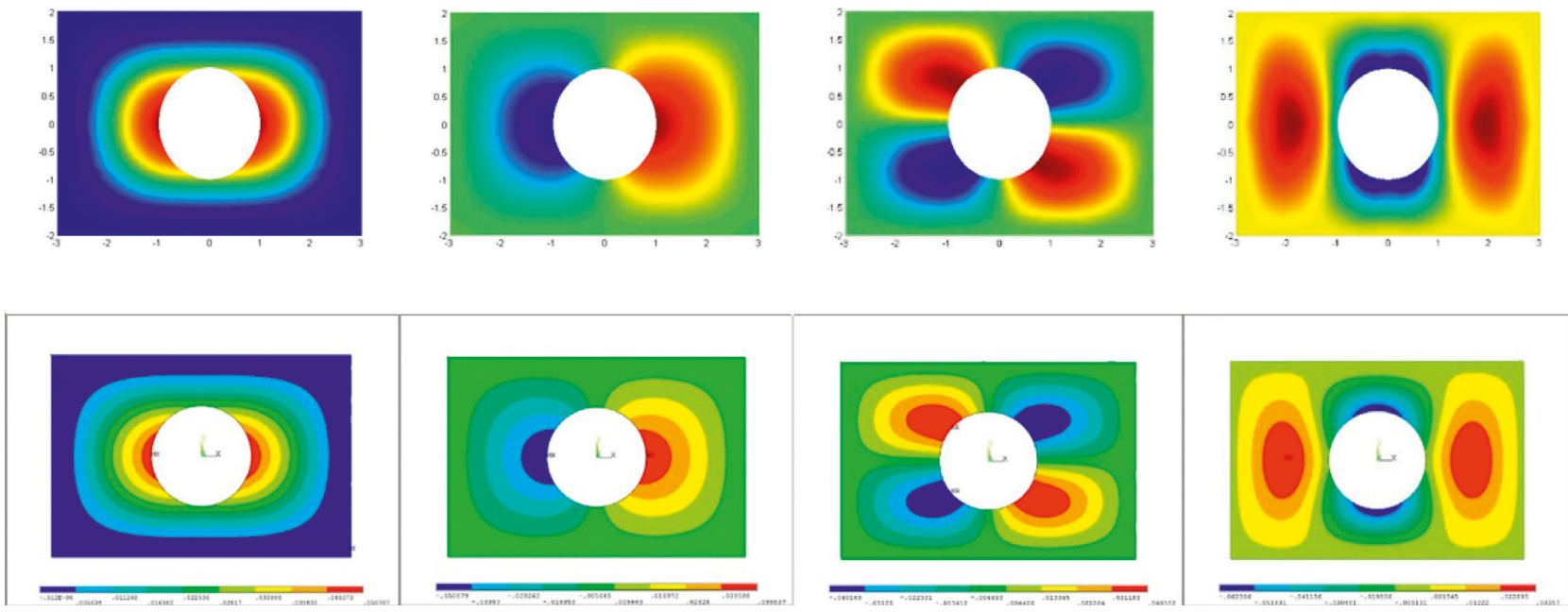

FIGURE 16: The corresponding modes of the first four orders of the rectangular plate with circular opening. 

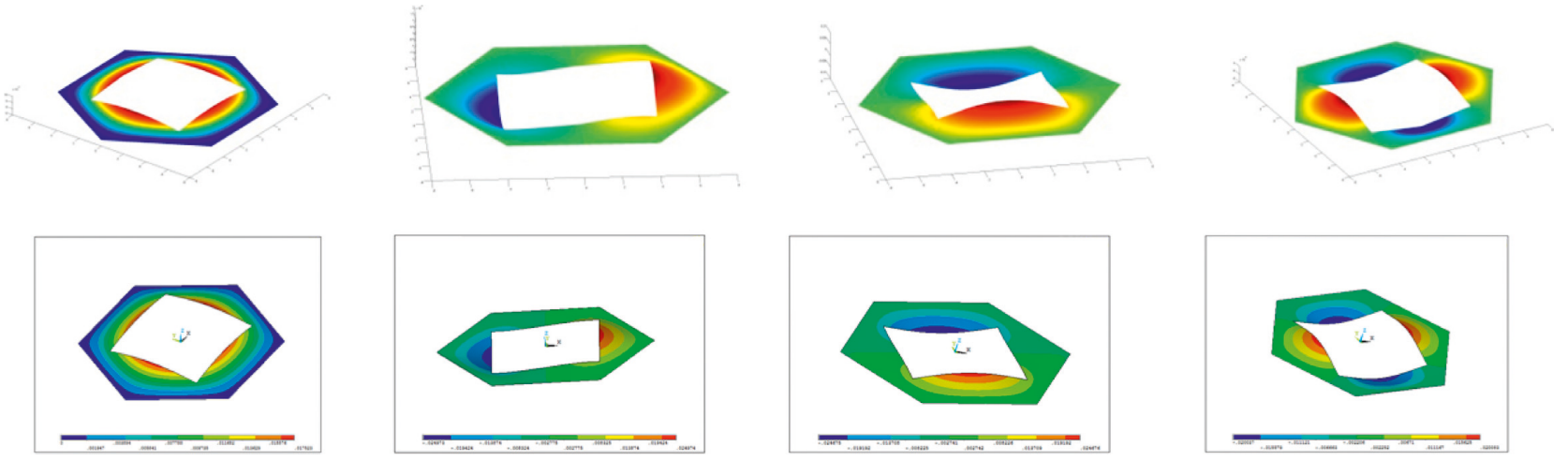

FIGURE 17: The corresponding modes of the first four orders of the hexagonal plate with rectangular opening.

$$
\begin{aligned}
V_{p}= & \frac{D}{2} \int_{0}^{a / 2} \int_{0}^{b / 2}\left[\left(\frac{\partial^{2} w}{\partial x^{2}}\right)^{2}+\left(\frac{\partial^{2} w}{\partial y^{2}}\right)^{2}\right. \\
& \left.+2 \mu\left(\frac{\partial^{2} w}{\partial x^{2}}\right)\left(\frac{\partial^{2} w}{\partial y^{2}}\right)+2(1-\mu)\left(\frac{\partial^{2} w}{\partial x \partial y}\right)^{2}\right] \mathrm{d} x \mathrm{~d} y .
\end{aligned}
$$

The bending strain energy of the circular opening is expressed as

$$
\begin{aligned}
V_{p r}= & \frac{D}{2} \int_{0}^{R} \int_{0}^{R}\left[\left(\frac{\partial^{2} w}{\partial x^{2}}\right)^{2}+\left(\frac{\partial^{2} w}{\partial y^{2}}\right)^{2}\right. \\
& \left.+2 \mu\left(\frac{\partial^{2} w}{\partial x^{2}}\right)\left(\frac{\partial^{2} w}{\partial y^{2}}\right)+2(1-\mu)\left(\frac{\partial^{2} w}{\partial x \partial y}\right)^{2}\right] \mathrm{d} x \mathrm{~d} y,
\end{aligned}
$$

where $D=E h^{3} / 12\left(1-\mu^{2}\right)$ and $E$ and $\mu$ are the material's Young's modulus and Poisson's ratio, respectively.

Kinetic energy of the rectangular plate without opening can be expressed as

$$
T=\frac{\rho h}{2} \int_{0}^{a / 2} \int_{0}^{b / 2}\left(\frac{\partial w}{\partial t}\right)^{2} \mathrm{~d} x \mathrm{~d} y .
$$

Open part's kinetic energy can be expressed as

$$
T_{r}=\frac{\rho h}{2} \int_{0}^{R} \int_{0}^{R}\left(\frac{\partial w}{\partial t}\right)^{2} \mathrm{~d} x \mathrm{~d} y,
$$

where $\rho$ is the density of material and $\omega$ is the circular frequency.

The elastic potential energy of the boundary springs can be expressed as

$$
\begin{aligned}
V_{s}= & \frac{1}{2} \int_{R}^{a / 2}\left[k_{y 0} w^{2}+K_{y 0}\left(\frac{\partial w}{\partial y}\right)^{2}\right]_{y=0} \mathrm{~d} x \\
& +\frac{1}{2} \int_{0}^{a / 2}\left[k_{y b} w^{2}+K_{y b}\left(\frac{\partial w}{\partial y}\right)^{2}\right]_{y=b / 2} \mathrm{~d} x \\
& +\frac{1}{2} \int_{R}^{b / 2}\left[k_{x 0} w^{2}+K_{x 0}\left(\frac{\partial w}{\partial y}\right)^{2}\right]_{x=0} \mathrm{~d} y \\
& +\frac{1}{2} \int_{0}^{b / 2}\left[k_{x a} w^{2}+K_{x a}\left(\frac{\partial w}{\partial y}\right)^{2}\right]_{x=a / 2} \mathrm{~d} y,
\end{aligned}
$$

where $k_{x 0}, k_{y 0}, k_{x b}$, and $k_{y b}$ are the displacement-constrained spring stiffness values in $x=0, y=0, x=a / 2$, and $y=b / 2$, respectively. $K_{x 0}, K_{y 0}, K_{x b}$, and $K_{y b}$ are the corner constrained spring stiffness values in $x=0, y=0, x=a / 2$, and $y=b / 2$, respectively.

Then, the total energy functional of the whole structure can be expressed as

$$
L=V_{p}-V_{p r}+V_{s}-T+T_{r}
$$

Table 14 shows the natural frequencies of free vibration of the rectangular plate with circular opening obtained by the present method and FEM. Figure 16 gives the corresponding modes of the first four orders.

Of course, the improved Rayleigh-Ritz method also can be applied to solve the vibration problems when the shape of the plate or the opening is changed as well. The corresponding vibration modes of the first four orders of hexagonal plate with rectangular opening are shown in Figure 17.

\section{Conclusion}

An improved Rayleigh-Ritz method is proposed to analyze the free vibration characteristic of the arbitrary-shaped plates under general boundary conditions. We expand the domain of admissible functions out of the structural domain to form a rectangular domain for which the admissible functions can be obtained easily. Besides, the spring model is adopted to simulate the general boundary conditions. The numerical results of the analytical solutions of rectangular, triangular, circular, oval, and curved trapezoidal thin plates are given. By comparing the natural frequencies and mode figures with the simulation results and reference examples, the remarkable convergence and accuracy of the present solution have repeatedly been demonstrated through the numerical examples. It is worth noting that, for a plate of symmetric shape, a quarter of the plate is adequate enough for the analysis to simplify the model. However, the symmetric and antisymmetric boundary conditions can be applied to the central cutting lines to get the frequencies and modes of the whole plate, and it is proved to be accurate.

As a semianalytical method, this method is used to calculate arbitrary-shaped thin plates under complicated 
boundary conditions. With unique advantages, the programming of the method is simple and easy to understand and of great compatibility. It should be highlighted that the present method can also be extended to the areas of other dynamic problems such as Mindlin plate, thick plate, and functionally gradient plate and shell.

\section{Data Availability}

All data included in this study are available from the corresponding author upon request.

\section{Conflicts of Interest}

The authors declare that they have no conflicts of interest.

\section{Acknowledgments}

The authors wish to express their gratitude to the National Natural Science Foundation of China (Contract No. 51878277).

\section{References}

[1] D. J. Dawe, "A finite element approach to plate vibration problems," Journal of Mechanical Engineering Science, vol. 7, no. 1, pp. 28-32, 1965.

[2] E. Viola, F. Tornabene, and N. Fantuzzi, "Generalized differential quadrature finite element method for cracked composite structures of arbitrary shape," Composite Structures, vol. 106, pp. 815-834, 2013.

[3] F. Tornabene, E. Viola, and N. Fantuzzi, "General higherorder equivalent single layer theory for free vibrations of doubly-curved laminated composite shells and panels," Composite Structures, vol. 104, pp. 94-117, 2013.

[4] C. W. Bert and M. Malik, "The differential quadrature method for irregular domains and application to plate vibration," International Journal of Mechanical Sciences, vol. 38, no. 6, pp. 589-606, 1996.

[5] C. W. Bert and M. Malik, "Differential quadrature: a powerful new technique for analysis of composite structures," Composite Structures, vol. 39, no. 3-4, pp. 179-189, 1997.

[6] M. A. Abdou and E. A. Khar-El din, "An infinite plate weakened by a hole having arbitrary shape," Journal of Computational and Applied Mathematics, vol. 56, no. 3, pp. 341-351, 1994.

[7] F. L. Liu and K. M. Liew, "Differential cubature method for static solutions of arbitrarily shaped thick plates," International Journal of Solids and Structures, vol. 35, no. 28-29, pp. 3655-3674, 1998.

[8] S. Ahmad, B. M. Irons, and O. C. Zienkiewicz, "Analysis of thick and thin shell structures by curved finite elements," International Journal for Numerical Methods in Engineering, vol. 2, no. 3, pp. 419-451, 1970.

[9] D. J. Gorman, "Free vibration analysis of the completely free rectangular plate by the method of superposition," Journal of Sound and Vibration, vol. 57, no. 3, pp. 437-447, 1978.

[10] N. Fantuzzi, F. Tornabene, and E. Viola, "Four-parameter functionally graded cracked plates of arbitrary shape: a GDQFEM solution for free vibrations," Mechanics of Advanced Materials and Structures, vol. 23, no. 1, pp. 89-107, 2016.
[11] K. Nagaya, "Simplified method for solving problems of vibrating plates of doubly connected arbitrary shape, part I: derivation of the frequency equation," Journal of Sound and Vibration, vol. 74, no. 4, pp. 543-551, 1981.

[12] K. Xie, M. Chen, L. Zhang, and D. Xie, "Wave based method for vibration analysis of elastically coupled annular plate and cylindrical shell structures," Applied Acoustics, vol. 123, pp. 107-122, 2017.

[13] S. Brischetto, "Convergence analysis of the exponential matrix method for the solution of 3D equilibrium equations for free vibration analysis of plates and shells," Composites Part B: Engineering, vol. 98, pp. 453-471, 2016.

[14] R. Li, P. Wang, R. Xue, and X. Guo, "New analytic solutions for free vibration of rectangular thick plates with an edge free," International Journal of Mechanical Sciences, vol. 131132, pp. 179-190, 2017.

[15] B. Mohammadzadeh and H. C. Noh, "Analytical method to investigate nonlinear dynamic responses of sandwich plates with FGM faces resting on elastic foundation considering blast loads," Composite Structures, vol. 174, pp. 142-157, 2017.

[16] E. Ruocco, V. Mallardo, V. Minutolo, and D. Di Giacinto, "Analytical solution for buckling of Mindlin plates subjected to arbitrary boundary conditions," Applied Mathematical Modelling, vol. 50, pp. 497-508, 2017.

[17] S. Abrate, "Vibration of point supported triangular plates," Computers \& Structures, vol. 58, no. 2, pp. 327-336, 1996.

[18] W. Karunasena and S. Kitipornchai, "Free vibration of sheardeformable general triangular plates," Journal of Sound and Vibration, vol. 199, no. 4, pp. 595-613, 1997.

[19] M. N. Pavlovic and F. C. Mbakogu, "Rayleigh estimates of the fundamental frequencies of vibration of circular plates," Journal of Sound and Vibration, vol. 198, no. 3, pp. 389-394, 1996.

[20] P. Kumari and A. K. Shakya, "Two-dimensional solution of piezoelectric plate subjected to arbitrary boundary conditions using extended Kantorovich method," Procedia Engineering, vol. 173, pp. 1523-1530, 2017.

[21] X. Wang, H. Chen, and J. Zhang, "An efficient boundary integral equation method for multi-frequency acoustics analysis," Engineering Analysis with Boundary Elements, vol. 61, pp. 282-286, 2015.

[22] W. L. Li, "Vibration analysis of rectangular plates with general elastic boundary supports," Journal of Sound and Vibration, vol. 273, no. 3, pp. 619-635, 2004.

[23] G. Jin, Z. Su, S. Shi, T. Ye, and S. Gao, “Three-dimensional exact solution for the free vibration of arbitrarily thick functionally graded rectangular plates with general boundary conditions," Composite Structures, vol. 108, pp. 565-577, 2014.

[24] Y. Kiani, "Shear buckling of FG-CNT reinforced composite plates using Chebyshev-Ritz method," Composites Part B: Engineering, vol. 105, pp. 176-187, 2016.

[25] Y. Kiani, "Thermal post-buckling of FG-CNT reinforced composite plates," Composite Structures, vol. 159, pp. 299306, 2017.

[26] W. L. Li, X. Zhang, J. Du, and Z. Liu, “An exact series solution for the transverse vibration of rectangular plates with general elastic boundary supports," Journal of Sound and Vibration, vol. 321, no. 1-2, pp. 254-269, 2009. 


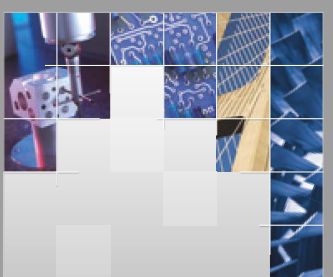

\section{Enfincering}
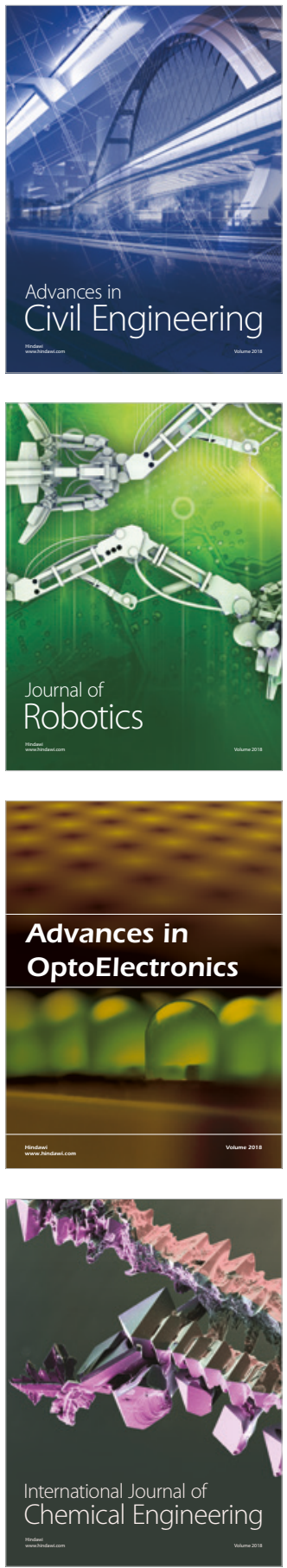

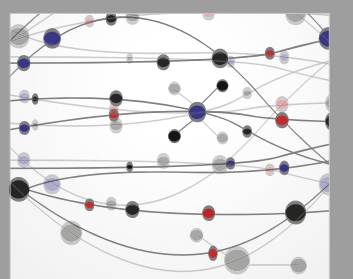

\section{Rotating \\ Machinery}

The Scientific World Journal

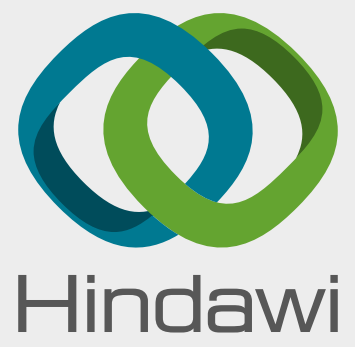

Submit your manuscripts at

www.hindawi.com
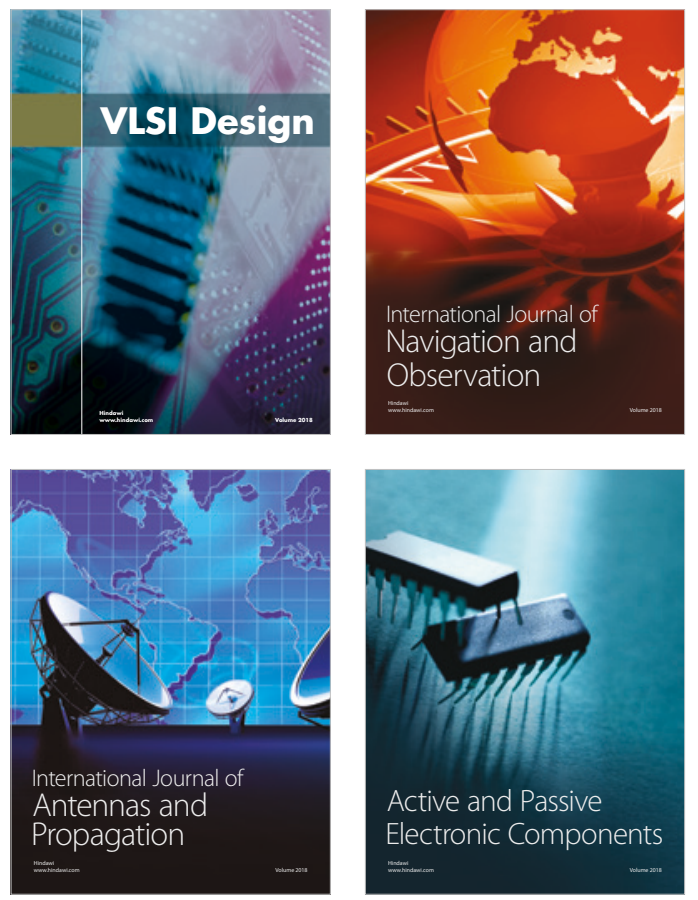
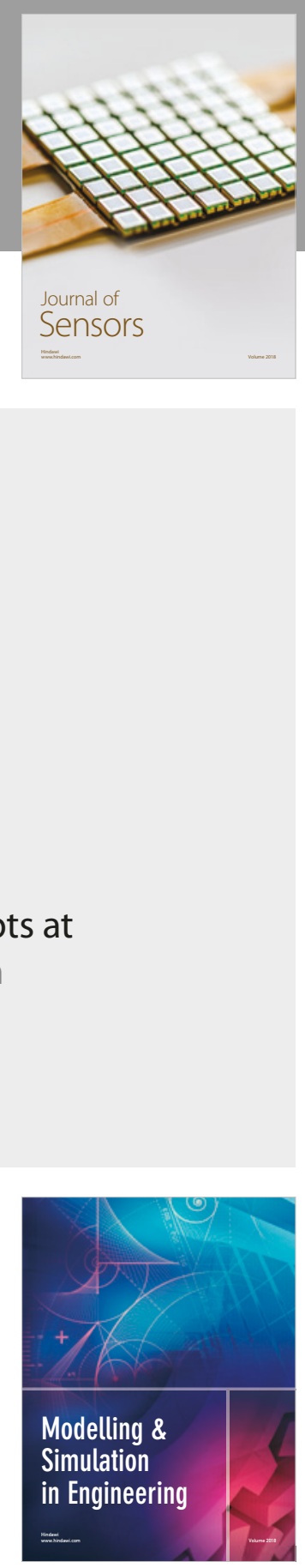

\section{Advances \\ Multimedia}
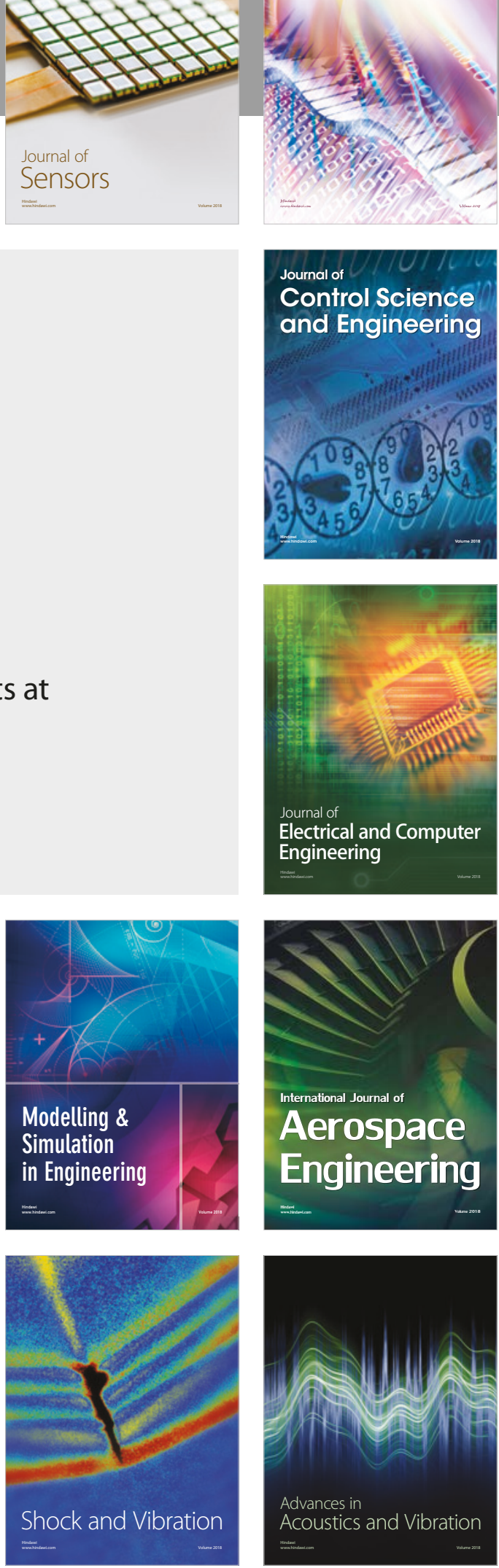